\title{
LOOP SPACES OF $H$-SPACES ${ }^{1}$
}

\author{
BY WILLIAM BROWDER
}

Communicated by Hans Samelson, June 6, 1960

Let $Y$ be an $H$-space (a space $Y$ with a continuous product $Y \times Y \rightarrow Y$ which has a unit element), $Y$ arcwise connected and simply connected, and let $X=\Omega Y$, the space of loops of $Y$ based at the unit. We will prove

THEOREM 1. If $H^{*}(X)$ (the singular cohomology ring over the integers) is a finitely generated module over the integers, then $X$ is of the same singular homotopy type as $K(G, 1)$ where $G$ is a free abelian group $\left(K(G, 1)=S^{1} \times \cdots \times S^{1}=\right.$ the $n$-torus, where rank of $\left.G=n\right)$.

Thus the loop space of an $H$-space $Y$ is infinite dimensional unless $Y=K(G, 2), G$ free abelian.

The proof depends on Theorem 2 below.

Let $p$ be a prime. Then the cohomology of an $H$-space $Y$ over $Z_{p}$ is a Hopf algebra (see [2]). If $\psi: Y \times Y \rightarrow Y$ is the multiplication in $Y$, then $\psi: H^{*}\left(Y ; Z_{p}\right) \rightarrow H^{*}\left(Y ; Z_{p}\right) \otimes H^{*}\left(Y ; Z_{p}\right)$ is the diagonal map of the Hopf algebra, the product being the cup product.

Let $A$ be a Hopf algebra over $Z_{p}, \psi: A \rightarrow A \otimes A$ the diagonal map, $\theta: A \otimes A \rightarrow A$ the product. An element $x \in A$ is called primitive if $\psi(x)=x \otimes 1+1 \otimes x$. An element $y \in A$ is called decomposable if $y \in \theta(\bar{A} \otimes \bar{A})$ where $\bar{A}$ is the subspace of $A$ consisting of positive dimensional elements. Let $P(A)$ denote the primitive elements of $A$, $D(A)$ the decomposable elements of $A, Q(A)=A / D(A)$. Let $\xi: A \rightarrow A$ be defined by $\xi(x)=x^{p}$. Then $\xi(A)$ is a Hopf subalgebra of $A$.

We quote a theorem of Milnor and Moore [9].

Theorem (Milnor AND Moore). Let $A$ be an associative, commutative Hopf algebra over $Z_{p}$ with $A_{0}=Z_{p}$. Then the sequence $0 \rightarrow P(\xi A)$ $\rightarrow^{f} P(A) \rightarrow^{o} Q(A)$ is exact.

Thus if $x \in P(A) \cap D(A)$, then $x=u^{p}$ for some $u \in A$.

Let $\rho^{i}$ denote the $i$ th Steenrod operation

$$
\mathcal{P}^{i}: H^{m}\left(X ; Z_{p}\right) \rightarrow H^{m+2 i(p-1)}\left(X ; Z_{p}\right) \quad \text { ( } p \text { an odd prime), }
$$

$S q^{i}$ denote the $i$ th Steenrod square

$$
S q^{i}: H^{m}\left(X ; Z_{2}\right) \rightarrow H^{m+i}\left(X ; Z_{2}\right) .
$$

${ }^{1}$ This note was written while the author was a National Science Foundation Postdoctoral Fellow. 
TheOREM 2. Let $Y$ be an $H$-space with $H^{*}\left(Y ; Z_{p}\right)$ $=P\left(y_{1}, \cdots, y_{n}, \cdots\right)=$ the ring of polynomials over $Z_{p}$ generated by $y_{1}, \cdots, y_{n}, \cdots$, with $\operatorname{dim} y_{i}$ even for all $i$. Let $x \in H^{2 m}\left(Y ; Z_{p}\right)$ be a primitive element. If $p \neq 2$ and $m=p^{r}+k$ with $0<k<p$, and $r>0$, then $\rho^{p^{r+i}}(x) \neq 0$ is indecomposable, $0 \leqq i<k$. If $p \neq 2$ and $1<m<p$ $\rho^{i}(x) \neq 0$ is indecomposable, $0<i<m$. If $p=2$, and $m=2^{r-1}+1$ with $r>1$, then $S q^{2^{r}}(x)$ is indecomposable.

Proof. Since $H^{*}\left(Y ; Z_{p}\right)$ is a polynomial ring $\rho^{m}(x)=x^{p} \neq 0$. Let $p \neq 2, m=p^{r}+k, k<p, r>1$. Then by the Adem relations [7]

$$
\mathcal{P}^{p^{r+k}}=k !\left(\mathcal{P}^{1}\right)^{k} \mathcal{P}^{p^{r}}
$$

so that $\mathcal{\rho}^{p^{r}+i}(x)=i !\left(\mathcal{P}^{1}\right)^{i} \mathcal{\odot}^{p^{r}}(x) \neq 0$ for $0 \leqq i \leqq k<p$. Now if $\alpha$ is an element of the Steenrod algebra and $Y$ is an $H$-space, then $\alpha\left(P\left(H^{*}\left(Y ; Z_{p}\right)\right) \subseteq \subseteq P\left(H^{*}\left(Y ; Z_{p}\right)\right)\right.$ since $\alpha$ is additive, so that $\mathbb{P}^{p^{r+i}}(x)$ is primitive in $H^{*}\left(Y ; Z_{p}\right)$. If $P^{p^{r+i}}(x)$ is decomposable then by the theorem of Milnor and Moore $\rho^{p^{r+i}}(x)=u^{p}$. But $\mathcal{P}^{1}\left(\mathcal{P}^{p^{r+i}}\right)$ $=(i+1) \rho^{p^{r+i+1}}$ so that if $i<k \rho^{1}\left(u^{p}\right) \neq 0$. But $\rho^{1}$ is a derivation by the Product Formula so that $\rho^{1}\left(u^{p}\right)=p u^{p-1}\left(\rho^{1} u\right)=0 \bmod p$. Hence $\mathbb{P}^{p^{r}+i}(x)$ is indecomposable in $H^{*}\left(Y ; Z_{p}\right)$.

If $1<m<p, p \neq 2$, we get from the Adem relations $p^{m}=m !\left(p^{1}\right)^{m}$, and we proceed similarly.

If $p=2$ and $m=2^{r-1}+1$ with $r>1$ we have that $x^{2}=S q^{2 r+2}(x)$ and from the Adem relations

$$
S q^{2^{r}+2}=S q^{2} S q^{2^{r}}+S q^{2^{r}+1} S q^{1} .
$$

Since $H^{*}\left(Y ; Z_{2}\right)$ is a polynomial ring on even dimensional generators $S q^{1} H^{*}\left(Y ; Z_{2}\right)=0$, for $S q^{1}$ changes dimension by 1 . Hence $S q^{2^{r+2}}(x)$ $=S q^{2} S q^{2^{r}}(x)$ so that $S q^{2^{r}}(x) \neq 0$ and is a primitive element. If $S q^{2^{r}}(x)$ $=u^{2}$ then $S q^{2}\left(u^{2}\right)=\left(S q^{2} u\right) u+\left(S q^{1} u\right)\left(S q^{1} u\right)+u\left(S q^{2} u\right)=0 \bmod 2$, since $S q^{1} \equiv 0$ in $H^{*}\left(Y ; Z_{2}\right)\left(S q^{2}\right.$ is a derivation on $\left.H^{*}\left(Y ; Z_{2}\right)\right)$. Hence $S q^{2^{r}}(x)$ is indecomposable. Q.E.D.

One can apply Theorem 2 to compute many Steenrod operations in the stable classical groups, using only the cohomology structure $\bmod p$ and the fact that the classifying space is an $H$-space. We will use Theorem 2 to prove Theorem 1 .

Proof of Theorem 1. Let $\bar{X}=$ the universal covering space of $X$. Then it follows from the results of [3] that $H^{*}(\bar{X})$ is finitely generated. Further, $\bar{X}=\Omega \bar{Y}$ where $\bar{Y}$ is the 2-connected fibre space over $Y$ (see [10]). Further $\bar{Y}$ is the fibre of a multiplicative fibre map of $Y$ into $K\left(\pi_{2}(Y), 2\right)$, and hence $\bar{Y}$ is an $H$-space. We will show that $\bar{X}$ is acyclic, (i.e., that $H^{i}(\bar{X})=0$ for $i>0$ ) and therefore $X$ is a $K(\pi, 1)$, 
finite dimensional with $\pi$ abelian finitely generated. Then $\pi$ must be free abelian and the result will be achieved.

Therefore we will assume that $\pi_{1}(X)=0$ and show that $X$ is acyclic.

If $X$ is not acyclic, then $H^{*}(X) /$ Torsion is nontrivial (see Part 1 of Theorem 3 of [4] or see [5]), and hence $H^{*}(X) /$ Torsion $=\Lambda\left(x_{1}, \cdots, x_{n}\right)$, the exterior algebra on odd dimensional generators $x_{1}, \cdots, x_{n}$ (see $\left.[2]\right)$. Since $H^{*}(X)$ is finitely generated, only a finite number of primes occur as torsion numbers of $H^{*}(X)$. Hence for almost all primes, in particular for all sufficiently large primes $p$, $H^{*}\left(X ; Z_{p}\right)=\left(H^{*}(X) /\right.$ Torsion $) \otimes Z_{p}$. Therefore we have $H^{*}\left(X ; Z_{p}\right)$ $=\Lambda\left(x_{1}, \cdots, x_{n}\right)$ (identifying $x_{i}$ with its image in $\left(H^{*}(X) /\right.$ Torsion) $\left.\otimes Z_{p}=H^{*}\left(X ; Z_{p}\right)\right)$ for all sufficiently large $p$.

By a theorem of Borel (Theorem 13.1 of [2]) we have that $H^{*}\left(Y ; Z_{p}\right)$ $=P\left(y_{1}, \cdots, y_{n}\right)$ if the prime $p$ is not a torsion number of $H(X)$, with $\operatorname{dim} y_{i}=\operatorname{dim} x_{i}+1$. Let the $y^{\prime}$ s be ordered so that $2 k=\operatorname{dim} y_{1}$ $\leqq \operatorname{dim} y_{i} \leqq \operatorname{dim} y_{n}=2 m, 1 \leqq i \leqq n$, and $k>1$ since $\operatorname{dim} x_{i}>2$ for all $i$.

Choose $p$ so large that $2 k+2(p-1)>2 m$ and $p>k>1$, or in other words choose $p>\max (m-k-1, k)$, and large enough that $p$ does not occur as a torsion number of $H^{*}(X)$. Then $y_{1}$ is primitive since it is in the first nonvanishing cohomology group of $Y$ and we may apply Theorem 2 to $y_{1} \in H^{*}\left(Y ; Z_{p}\right)$. Hence $\rho^{1}\left(y_{1}\right) \neq 0$ and is an indecomposable element in $H^{*}\left(Y ; Z_{p}\right)$. But $\operatorname{dim} \rho^{1}\left(y_{1}\right)=2 k+2(p-1)$ $>2 m$, and all elements of $H^{q}\left(Y ; Z_{p}\right)$ are decomposable if $q>2 m$. This is a contradiction, so $X$ is acyclic. Q.E.D.

One might conjecture that if $X$ is a homotopy commutative $H$ space and $H^{*}(X)$ is finitely generated then $X$ is of the same singular homotopy type as $K(G, 1)$ with $G$ a free abelian group. Araki, James and Thomas have shown that the usual multiplication on a compact Lie group $G$ is not homotopy commutative unless $G$ is a torus [1], and James [8] has shown that the spheres $S^{3}$ and $S^{7}$ have no homotopy commutative multiplications. It will be shown elsewhere [6] that if $X$ is a homotopy commutative $H$-space with $H^{*}(X)$ finitely generated, then $H^{*}(X)$ has no 2-torsion. Hence the Lie groups which have 2-torsion (such as $S O(n)$ and the exceptional groups) have no homotopy commutative multiplications on them. It will also be shown in [6] that if $X$ is homotopy associative and homotopy commutative, and $H^{*}(X)$ is finitely generated, then $H^{*}(X)$ has no torsion, so that $H^{*}(X)=\Lambda\left(x_{1}, \cdots, x_{n}\right)$.

In conclusion, it is pleasant to acknowledge the value of some conversations with J. Stasheff. 


\section{BIBLIOGRAPHY}

1. S. Araki, I. M. James, and Emery Thomas, Homotopy-Abelian Lie groups, Bull. Amer. Math. Soc. vol. 66 (1960) pp. 332-334.

2. A. Borel, Sur la cohomologie des espaces fibrés principaux et des espaces homogènes de groupes de Lie compacts, Am. of Math. vol. 57 (1953) pp. 115-207.

3. W. Browder, The cohomology of covering spaces of $H$-spaces, Bull. Amer. Math. Soc. vol. 65 (1959) pp. 140-141.

4. - Homology and homotopy of H-spaces, Proc. Nat. Acad. Sci. U.S.A. vol. 46 (1960) pp. 543-545.

5. - Torsion in $H$-spaces, to appear.

6. - Homotopy commutative H-spaces, to appear.

7. H. Cartan, Sur l'itérations des operations de Steenrod, Comment. Math. Helv. vol. 29 (1955) pp. 40-58.

8. I. M. James, Multiplication on spheres (I), Proc. Amer. Math. Soc. vol. 8 (1957) pp. 192-196.

9. J. W. Milnor and J. C. Moore, On the structure of Hopf algebras, to appear.

10. J.-P. Serre, Groupes d'homotopie et classes de groupes abeliens, Ann. of Math. vol. 58 (1953) pp. 258-294.

CORNELl University AND

The Mathematical Institute, Oxford 